(1)

CrossMark

\title{
Bacteria-driven peribronchial lymphoid neogenesis in bronchiectasis and cystic fibrosis
}

\author{
Justine Frija-Masson 1,2,3 Clémence Martin $^{1,2,3}$, Lucile Regard ${ }^{1,2,3}$, \\ Marie-Noëlle Lothe ${ }^{1,2,3}$, L'housseine Touqui ${ }^{3}$, Aurélie Durand ${ }^{1,4,5}$, Bruno Lucas ${ }^{1,4,5}$, \\ Diane Damotte ${ }^{1,6}$, Marco Alifano $^{1,7}$, Isabelle Fajac ${ }^{1,3,8}$ and Pierre-Régis Burgel ${ }^{1,2,3^{\prime}}$ \\ Affiliations: ${ }^{1}$ Université Paris Descartes, Sorbonne Paris Cité, Paris, France. ${ }^{2}$ Service de Pneumologie, \\ Hôpital Cochin, AP-HP, Paris, France. ${ }^{3}$ Paris Descartes University/Pasteur Institute Joint Research Unit, \\ Pathobiology of Cystic Fibrosis and Chronic Airway Diseases, UPRES EA2511, Paris, France. ${ }^{4}$ INSERM U1016, \\ Institut Cochin, Paris, France. ${ }^{5}$ Centre National de la Recherche Scientifique, Unité Mixte de Recherche 8104, \\ Paris, France. ${ }^{6}$ Service d'Anatomie Pathologique, Hôpital Cochin, AP-HP, Paris, France. ${ }^{7}$ Service de Chirurgie \\ Thoracique, Hôpital Cochin, AP-HP, Paris, France. ${ }^{8}$ Service de Physiologie et Explorations Fonctionnelles, \\ Hôpital Cochin, AP-HP, Paris, France.
}

Correspondence: Pierre-Régis Burgel, 27 rue du Faubourg Saint Jacques, 75014 Paris, France.

E-mail: pierre-regis.burgeldaphp.fr

@ERSpublications

Bacterial infection-induced peribronchial lymphoid neogenesis in bronchiectasis and CF may involve airway epithelium http://ow.ly/pDMB308a3B0

Cite this article as: Frija-Masson J, Martin C, Regard L, et al. Bacteria-driven peribronchial lymphoid neogenesis in bronchiectasis and cystic fibrosis. Eur Respir J 2017; 49: 1601873 [https://doi.org/10.1183/ 13993003.01873-2016].

ABSTRACT We aimed to characterise lymphoid neogenesis in bronchiectasis and cystic fibrosis (CF) lungs and to examine the role of bacterial infection.

Lymphoid aggregates were examined using immunohistochemical staining and morphometric analysis in surgical lung sections obtained from nonsmokers and patients with bronchiectasis or CF. Sterile, Pseudomonas aeruginosa- or Staphylococcus aureus-coated agarose beads were instilled intratracheally in mice. Kinetics of lymphoid neogenesis and chemokine expression were examined over 14 days.

Lymphoid aggregates were scarce in human lungs of nonsmokers, but numerous peribronchial lymphoid aggregates containing B-lymphocytes, T-lymphocytes, germinal centres and high endothelial venules were found in bronchiectasis and CF. Mouse lungs contained no lymphoid aggregate at baseline. During persistent $P$. aeruginosa or $S$. aureus airway infection peribronchial lymphoid neogenesis occurred. At day 14 after instillation, lymphoid aggregates expressed markers of tertiary lymphoid organs and the chemokines CXCL12 and CXCL13. The airway epithelium was an important site of CXCL12, CXCL13 and interleukin-17A expression, which began at day 1 after instillation.

Peribronchial tertiary lymphoid organs are present in bronchiectasis and in CF, and persistent bacterial infection triggered peribronchial lymphoid neogenesis in mice. Peribronchial localisation of tertiary lymphoid organs and epithelial expression of chemokines suggest roles for airway epithelium in lymphoid neogenesis.

This article has supplementary material available from erj.ersjournals.com

Received: Sept 222016 | Accepted after revision: Jan 042017

Support statement: The research leading to these results has received funding from the European Union's Seventh Framework Programme (FP7/2007-2013) under grant agreement number 603038 (CFMATTERS), from the Chancellerie de l'Université de Paris (Legs Poix) and the French cystic fibrosis association (Vaincre la Mucoviscidose). J. Frija-Masson was the recipient of a fellowship grant provided by "Fondation Groupama" for rare diseases. Funding information for this article has been deposited with the Crossref Funder Registry.

Conflict of interest: Disclosures can be found alongside this article at erj.ersjournals.com

Copyright @ERS 2017 


\section{Introduction}

Chronic bacterial infection is the hallmark of lung disease in patients with bronchiectasis [1] and in those with cystic fibrosis (CF) [2]. Bacterial infection by Gram-negative (e.g. Pseudomonas aeruginosa) and/or Gram-positive (e.g. Staphylococcus aureus) promotes airway inflammation and structural damage, contributing to disease progression in both diseases [3, 4]. Host-pathogen interaction studies in these diseases have often focused on innate immune responses, involving airway epithelial responses and recruitment of phagocytes (e.g. neutrophils and macrophages) [3, 5]. However, bronchial biopsies obtained in patients with bronchiectasis showed a subepithelial infiltrate that was mostly composed of mononuclear cells, which were characterised as being predominantly T-lymphocytes [6, 7]. Moreover, a study of bronchial biopsies in CF children has shown that in contrast to the neutrophil-dominated inflammation present in the airway lumen, lymphocytes accumulates in the bronchial mucosa [8]. These data suggest that the adaptive immune response contributes to the pathophysiology of bronchiectasis and CF.

The adaptive immune response is initiated in secondary lymphoid organs, such as lymph nodes and the spleen, in which T- and B-lymphocytes clonally expand and are then recruited to the site of inflammation [9]. Secondary lymphoid organs develop during embryogenesis, but ectopic lymphoid structures, exhibiting structural similarities with secondary lymphoid organs (and called tertiary lymphoid organs) can develop at the mucosal surface after birth [9-11]. Tertiary lymphoid organs are not present in normal lungs [12], but have been described in the lungs of subjects with chronic airway diseases including asthma [13] and chronic obstructive pulmonary disease [14], and in lung cancer [15, 16]. Although lymphoid aggregates have long been described in some patients with bronchiectasis [17] and in patients with severe CF [18], it remains unknown whether these aggregates are organised into tertiary lymphoid organs, containing distinct B- and T-cell areas, and germinal centres in which antigen-specific adaptive immune responses can be initiated $[10,19]$.

Animal studies have shown that pulmonary tertiary lymphoid organs can be induced by stimuli relevant to chronic airway diseases, including allergens [20], cigarette smoke [21], viral infection [22] and repeated airway challenges with bacterial products $[23,24]$ or heat-inactivated bacteria [25]. However, it remains unclear whether chronic bacterial infection results in the development of tertiary lymphoid organs, a process called lymphoid neogenesis. Mechanisms leading to lymphoid neogenesis appear to differ among stimuli: antigen- [20], cigarette smoke- [26] and virus-induced [25] lymphoid neogenesis appeared dependent on the chemokine CXCL13, whereas heat-inactivated $P$. aeruginosa-induced lymphoid neogenesis occurred in the absence of CXCL13 and required the chemokine CXCL12 [25]. Finally, the development of tertiary lymphoid organs in the lung depends on interleukin (IL)-17A produced by T-cells $[25,27]$.

In the present study, lymphocytic infiltration was characterised in the lungs of patients with bronchiectasis or with CF, compared with control nonsmokers. Peribronchial lymphoid aggregates exhibiting the characteristics of tertiary lymphoid organs were present in patients with bronchiectasis and in those with $\mathrm{CF}$. Because bronchiectasis and CF are characterised by chronic airway infection, we hypothesised a role for infection in lymphoid neogenesis. Therefore, we examined the effects of persistent airway infection by Gram-negative ( $P$. aeruginosa) or Gram-positive ( $S$. aureus) bacteria on lymphocyte recruitment and lymphoid neogenesis in mouse airways.

\section{Methods}

Human lung specimens

Human lung specimens were obtained at surgery from 17 patients who underwent lung resection for solitary lung cancer (controls), from eight patients who underwent lobar resection for localised bronchiectasis (six post-tuberculosis, one sequelae of childhood pneumonia and one idiopathic) and from 17 adult CF patients at transplantation. All patients were nonsmokers and no bacteria was identified in cultures of bronchial aspirates obtained in control patients. Clinical characteristics of patients are shown in online supplementary table S1. In controls, only one block of tissue removed in a macroscopically normal area at a distance from the cancer was available. In patients with bronchiectasis or CF, tissues were obtained from two to three different tissue blocks, whose locations were chosen randomly, to reduce the potential sampling bias related to irregular distribution of tissue remodelling. The study obtained agreement of the local ethics committee (CPP Ile de France II; number 1072) for the use of samples of human origin.

\section{Animal studies \\ Agarose bead model of persistent airway infection}

All animal experiments received approval from the ethical review board of Paris Descartes University (CEEA34.PRB.135.12). Female C57BL/6N mice aged 10 weeks were purchased from Harlan (Gannat, France) and housed in conventional conditions. Persistent airway infection was obtained by intratracheal instillation of agarose beads containing $P$. aeruginosa $\mathrm{O} 1$ (PAO1; $5 \times 10^{5} \mathrm{CFU}$ per animal) or S. aureus 
(SA; a methicillin-sensitive clinical strain isolated from the sputum of a CF patient; $10^{7} \mathrm{CFU}$ per animal), following the method described by MARTIN et al. [28]. Mice were instilled with sterile, PAO1-or SA-coated beads and sacrificed at day 1, 4, 7 or 14 after instillation.

\section{Immunohistochemical staining}

Lungs were removed after flushing $4 \%$ paraformaldehyde through the right heart, and fixed in $4 \%$ paraformaldehyde. 5- $\mu \mathrm{m}$ paraffin sections obtained in human or in mouse lungs were immunostained as described previously [29] for detecting B-lymphocytes $\left(\mathrm{CD}^{2} 0^{+}\right)$, T-lymphocytes $\left(\mathrm{CD}^{+}\right)$, high endothelial venules ( peripheral node adressin $\left(\mathrm{PNAd}^{+}\right)$), follicular dendritic cells, proliferating cell nuclear antigen (PCNA; for the identification of germinal centres [30]) and for CXCL12, CXCL13 and IL-17A. A detailed description of the sources of primary antibodies, dilutions and unmasking techniques is provided in online supplementary table S2. Biotinylated antimouse, antirabbit, antirat or antigoat antibodies (dilution 1:200; Vector Laboratories, Burlingame, CA, USA) were used for secondary antibodies and bound antibodies were visualised according to standard protocols for avidin-biotin-peroxidase complex method (Elite ABC kit; Vector Laboratories). Tissue sections were counterstained with haematoxylin or methyl green (Vector Laboratories). No staining was observed when primary antibodies were omitted (online supplementary figure S1).

\section{Morphometric analyses}

In both human and animal studies, quantitative morphometric analysis was performed by two independent observers using a light microscope (Leica Microsystems, Wetzlar, Germany) connected to a computer [29]. A lymphoid aggregate was defined as a $\mathrm{CD}_{2} 0^{+}$or $\mathrm{CD}^{+}$cell aggregate that could be detected using a low-power field $(\times 16)$. The number of lymphoid aggregates was expressed by $\mathrm{cm}^{2}$ of lung. Volume ratios of epithelium stained for CXCL12, CXCL13 and IL-17A were determined by point counting, as described previously [29, 31].

\section{Flow cytometry}

Bronchoalveolar lavage was performed by flushing $2 \mathrm{~mL}$ of saline in mouse lungs and bronchoalveolar lavage fluids (BALFs) were filtered through a $40-\mu \mathrm{m}$ nylon membrane to eliminate agarose beads. BALFs were centrifuged at $250 \times g$ for $6 \mathrm{~min}$ at $4^{\circ} \mathrm{C}$. The cell pellet was suspended in $200 \mu \mathrm{L}$ of PBS containing fetal calf serum and separated into two equal parts for assessment of myeloid and lymphoid staining by flow cytometry, as described previously [32]. Characterisation of haematopoietic cell subsets was performed as described in a previous report [33] (online supplementary table S3), and a description of primary antibodies is provided in online supplementary table S4.

\section{Measurement of CXCL12, CXCL13 and IL-17A protein in lung homogenates}

CXCL12, CXCL13 and IL-17A protein concentrations in mouse lung homogenates were determined using ELISA kits (R\&D Systems, Abingdon, UK), according to the manufacturer's recommendations.

\section{Statistics}

Data obtained from morphometric analysis were analysed using the nonparametric Kruskal-Wallis test followed by the post hoc Dunn's test. The interobserver coefficients of variation for morphometric measurements were $<15 \%$. Data obtained from measurements of protein concentrations in lung homogenates were analysed using one-way ANOVA for repeated measurements followed by the post hoc Student-Neuman-Keuls test for multiple comparisons. All analyses were performed using Prism 5 software (GraphPad, La Jolla, CA, USA). p-values $\leqslant 0.05$ were considered statistically significant.

\section{Results}

Peribronchial lymphoid aggregates in human lungs

In the lungs of control nonsmokers, $\mathrm{CD} 20^{+}$lymphoid aggregates were scarce and small, and did not contain germinal centres; they were localised in perivascular or in alveolar space at a distance from the bronchi. In the lungs of patients with bronchiectasis or with $\mathrm{CF}$, numerous large $\mathrm{CD} 20^{+}$lymphoid aggregates were present, and were predominantly localised adjacent to bronchi. In the lungs of patients with bronchiectasis or CF many lymphoid aggregates showed characteristics of tertiary lymphoid organs with distinct B-cell and T-cell areas, high endothelial venules and germinal centres (characterised by positive staining for follicular dendritic cells (FDC) and PCNA). Representative photomicrographs are shown in figure $1 \mathrm{a}$ and $\mathrm{b}$. Quantitative morphometric analysis confirmed markedly increased numbers of $\mathrm{CD}_{2}{ }^{+}, \mathrm{CD}^{+}, \mathrm{FDC}^{+}, \mathrm{PCNA}^{+}$and $\mathrm{PNAd}^{+}$lymphoid aggregates in the lung of patients with bronchiectasis or with $\mathrm{CF}$ (figure $1 \mathrm{c}-\mathrm{g}$ ). 
a)

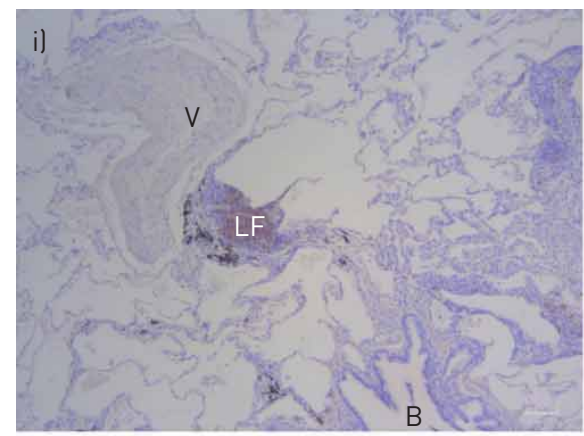

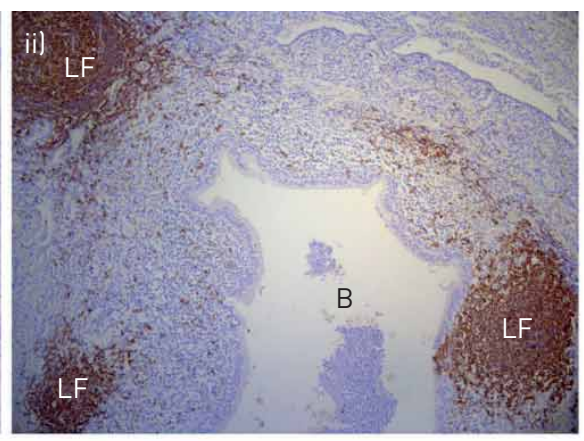

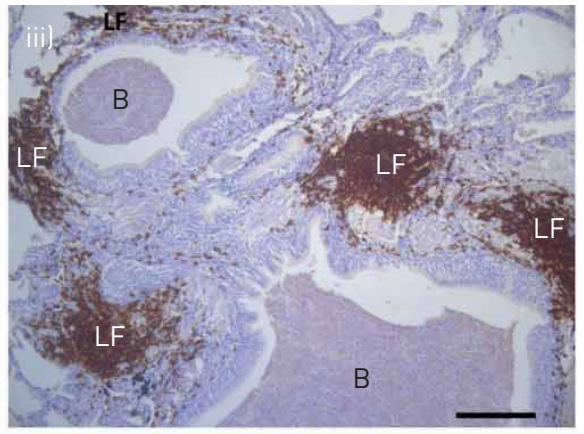

b)
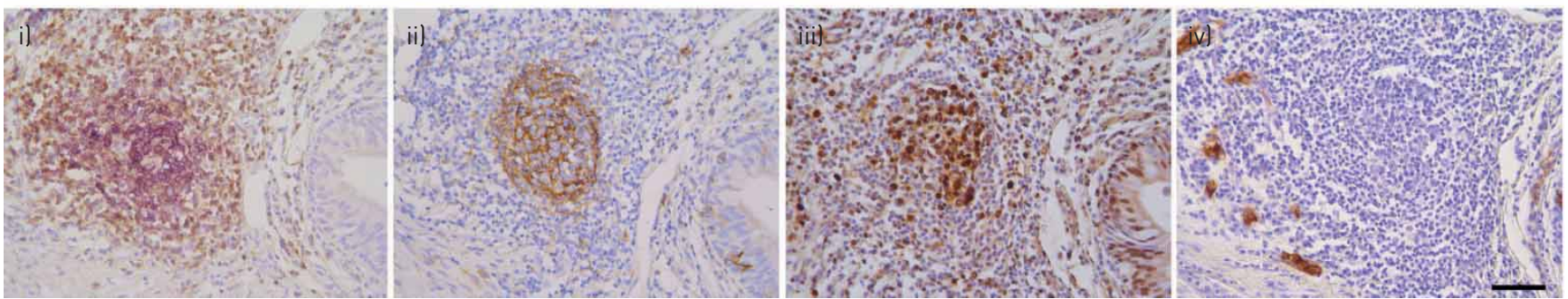
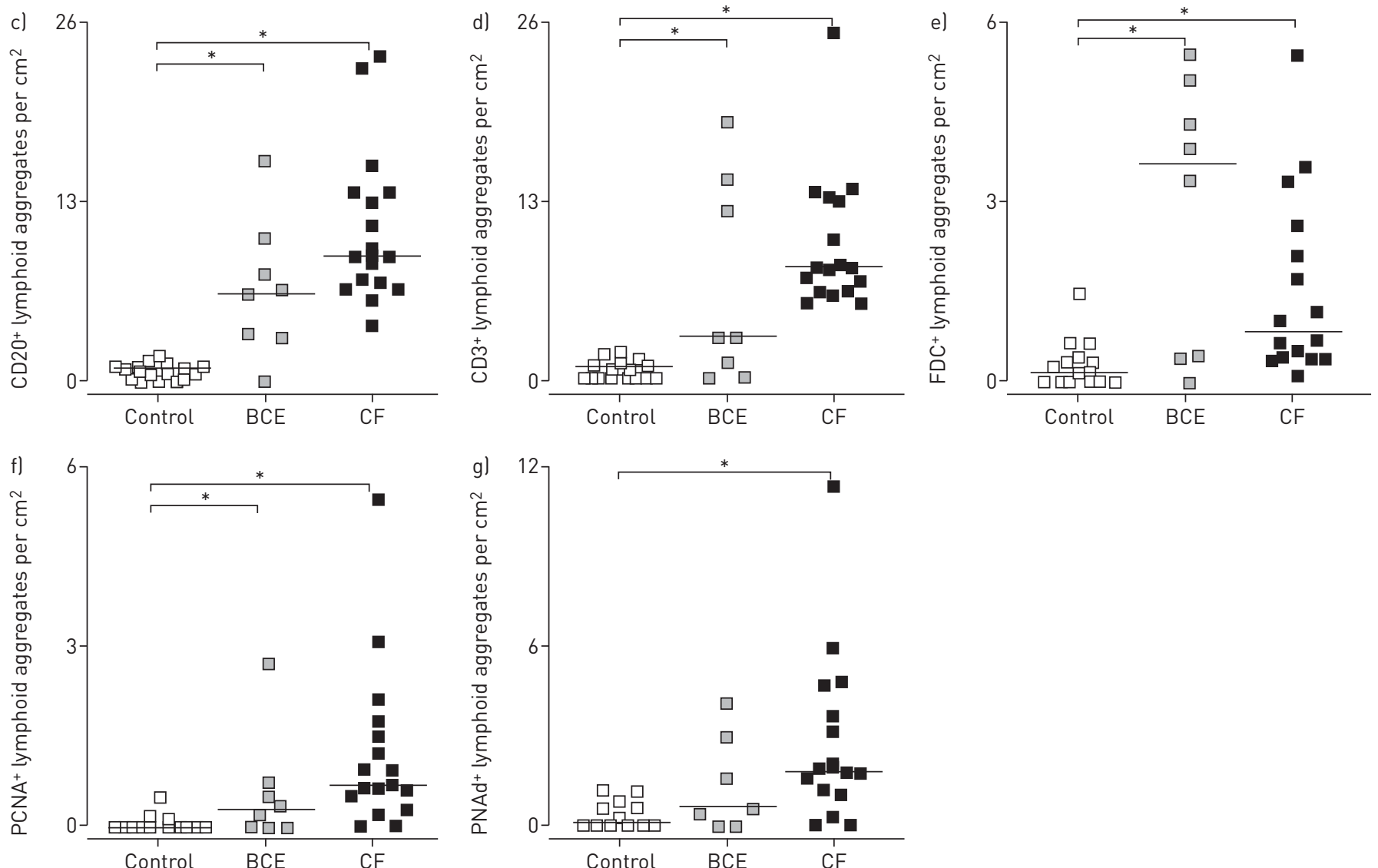

FIGURE 1 Peribronchial lymphoid aggregates in human lungs. a) Representative photomicrographs of CD20 immunostainings. In i) control subjects, only small and scarce CD20 lymphoid aggregates (LF; brown) were found and localised at a distance from the bronchi (B), often close to blood vessels (V). In patients with ii) bronchiectasis (BCE) and iii) cystic fibrosis (CF), numerous large $\mathrm{CD}_{20} \mathrm{O}^{+}$lymphoid aggregates were present and were localised immediately adjacent to bronchi, underneath the airway epithelium. Scale bar $=200 \mu \mathrm{m}$. b) Representative photomicrographs of the characterisation of a CD20 lymphoid aggregate as a tertiary lymphoid organ in the lung of a CF patient. Tissue sections were double-stained for i) B-lymphocytes (CD20; red) and T-lymphocytes (CD3; brown) and adjacent sections were stained for ii) follicular dendritic cells (FDC; brown), iii) germinal centres (proliferating cell nuclear antigen (PCNA); brown) and iv) high endothelial venules (peripheral node adressin (PNAd); brown). Scale bar $=50 \mu \mathrm{m}$. Morphometric quantification of cl $\mathrm{CD}^{+} \mathrm{O}^{+}$, d) $\mathrm{CD}^{+}$, e) $\mathrm{FDC}^{+}$, fl $\mathrm{PCNA}^{+}$and g) PNAd lymphoid aggregates. Adjacent tissue sections were immunostained for CD20, CD3, FDC, PCNA and PNAd. Lymphoid aggregates with positive staining for CD20, CD3, FDC, PCNA and PNAd were counted and total lung surface was measured as described in the methods section. The number of $\mathrm{CD}^{2} \mathrm{O}^{+}, \mathrm{CD}^{+}, \mathrm{FDC}^{+}, \mathrm{PCNA} \mathrm{A}^{+}$and $\mathrm{PNAd}^{+}$lymphoid aggregates was significantly higher in subjects with BCE and CF compared with controls. Each symbol represents data obtained from one patient. Horizontal bars represent medians. ${ }^{*}$ : $p<0.05$ compared with controls. 
Expression of the chemokines CXCL12 and CXCL13 in human lungs

In the lungs of control nonsmokers, immunostaining for CXCL12 and CXCL13 was weak and localised in the airway epithelium. In bronchiectasis and in CF lungs, CXCL12 and CXCL13 immunostaining was present in peribronchial lymphoid aggregates. Additionally, airway epithelium strongly stained for CXCL12 and CXCL13 in ciliated, but not in goblet cells (online supplementary figure S2). IL-17A immunostaining was weak in the epithelium of control nonsmokers, but was increased in the epithelium of subjects with bronchiectasis or CF. Representative photomicrographs are shown in figure $2 \mathrm{a}$ and morphometric analysis of CXCL12, CXCL13 and IL-17A staining in airway epithelium is shown in figure 2b-d.

\section{Persistent airway infection induces peribronchial lymphoid neogenesis in mice}

Next, we examined the effect of persistent bacterial infection on lymphoid neogenesis in mice. A single intratracheal instillation of agarose beads containing PAO1 or SA resulted in bacterial infection that persisted for 14 days (online supplementary figure S3). At day 14 after instillation of PAO1- or SA-coated beads, lymphoid aggregates containing $\mathrm{CD} 20^{+} \mathrm{B}$-cells and $\mathrm{CD}^{+} \mathrm{T}$-cells, CD21 ${ }^{+} \mathrm{FDCs} \mathrm{PNAd}^{+}$high endothelial venules and $\mathrm{PCNA}^{+}$proliferating B-cells were found adjacent to bead-containing (infected) airways. However, no lymphoid aggregate was found in morphologically normal lung at a distance from these airways. Similarly, no lymphoid aggregate was found in the airways of mice instilled with sterile beads or in those of control (no instillation) mice. Representative photomicrographs are shown in figure 3.

To examine the role of persistent versus acute infection on lymphoid neogenesis, we instilled mice intranasally with sublethal doses of PAO1 $\left(10^{6} \mathrm{CFU}\right.$ per animal) or SA (109 $\mathrm{CFU}$ per animal) without embedding the bacteria in agarose beads. At 14 days after instillation, PAO1 or SA infection were absent and no lymphoid aggregates were found (not shown), confirming the requirement for persistent airway infection for inducing peribronchial lymphoid neogenesis.

\section{Kinetics of bacteria-induced pulmonary lymphoid neogenesis in mice}

Recruitment of myeloid and lymphoid cells in airways during persistent bacterial infection were first assessed by flow cytometry in BALF of mice instilled with PAO1-coated beads (figure 4). Early response to PAO1 infection involved massive recruitment of granulocytes within $24 \mathrm{~h}$ of instillation, but persistent infection was associated with a subsequent decrease in total number of cells in BALF. At day 4 post-instillation, decrease in the number of neutrophils was associated with recruitment of other innate immune cells, including macrophages, monocytes and natural killer cells. Airway recruitment of myeloid dendritic cells and adaptive immune cells, i.e. B- and T-lymphocytes began between day 4 and day 7 and was increased at day 14 after instillation. Visual cell counts in BALF from SA-infected mice showed comparable results, whereas instillation of sterile beads resulted in transient and moderate increase in BALF macrophages, without changes in neutrophil or lymphocyte numbers (online supplementary figure S4).

Tissue recruitment of lymphocytes was then analysed by immunohistochemical staining. Within $24 \mathrm{~h}$ of instillation of PAO1- or SA-coated beads, CD20 ${ }^{+}$B-cells were recruited adjacent to the epithelium of bead-containing airways; during the first week of infection, elongated $\mathrm{CD} 20^{+}$lymphoid aggregates were found adjacent to bead-containing airways. Infection-induced recruitment of $\mathrm{CD}^{+} \mathrm{T}$-cells was delayed: $\mathrm{CD}^{+}$lymphoid aggregates were first identified at day 7 after instillation of PAO1- or SA-coated beads and peaked at day 14. Morphometric analysis of the numbers of $\mathrm{CD}_{2} 0^{+}$and $\mathrm{CD}^{+}$lymphoid aggregates over 14 days after intratracheal instillation of PAO1- or SA-coated beads are presented in figure 5. Representative photomicrographs are shown in online supplementary figure S5. Tertiary lymphoid organs persisted $\geqslant 28$ days after instillation (not shown).

Kinetics of bacteria-induced pulmonary expression of CXCL12, CXCL13 and IL-17A in mice In control (no instillation) mice and in mice instilled with sterile agarose beads, CXCL12, CXCL13 and IL17A protein were detectable in lung homogenates (figure $6 \mathrm{a}-\mathrm{c}$ ); only weak immunohistochemical staining was found in airway epithelium (not shown).

At day 1 after instillation of PAO1-coated beads, CXCL13 and IL-17A, but not CXCL12 protein contents were increased in total lung homogenates. IL-17A contents in lung homogenates remained increased over 14 days of infection, whereas CXCL13 contents decreased at days 4 and 14 .

Immunohistochemical staining showed that airway epithelium was an important site of CXCL12, CXCL13 and IL-17A expression and morphometric analysis showed that CXCL12, CXCL13 and IL-17A were significantly increased in epithelium at day 1 after infection (figure $6 \mathrm{~d}-\mathrm{f}$ ). Immunostaining showed that epithelial CXCL13 and IL-17A remained increased over the 14 days of infection, whereas epithelial CXCL12 decreased during persistent infection. CXCL12 and CXCL13, but not IL-17A were also expressed in lymphoid aggregates at day 14 after PAO1 or SA infection (representative photomicrographs are shown in figure $6 \mathrm{~g}$ ). 
Controls
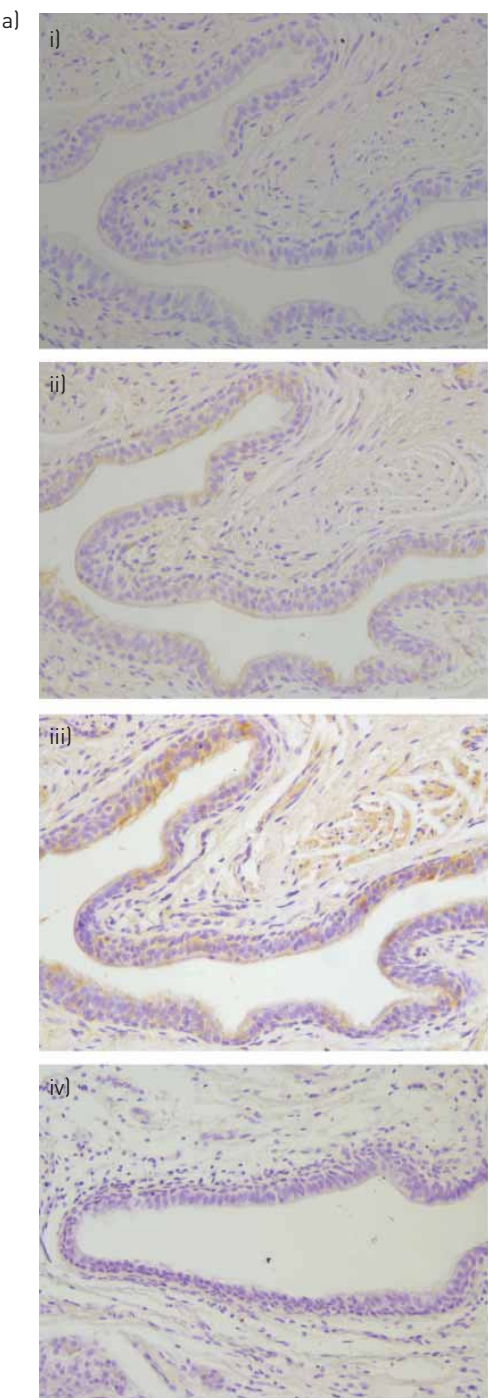

b)

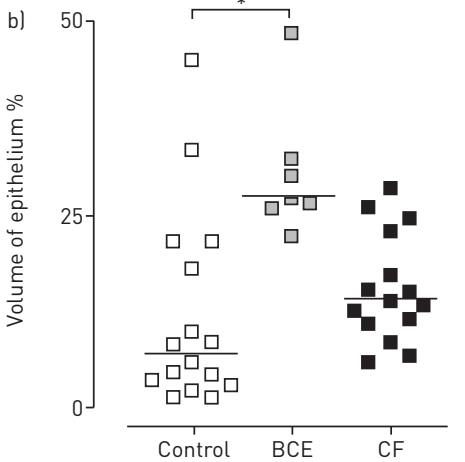

BCE
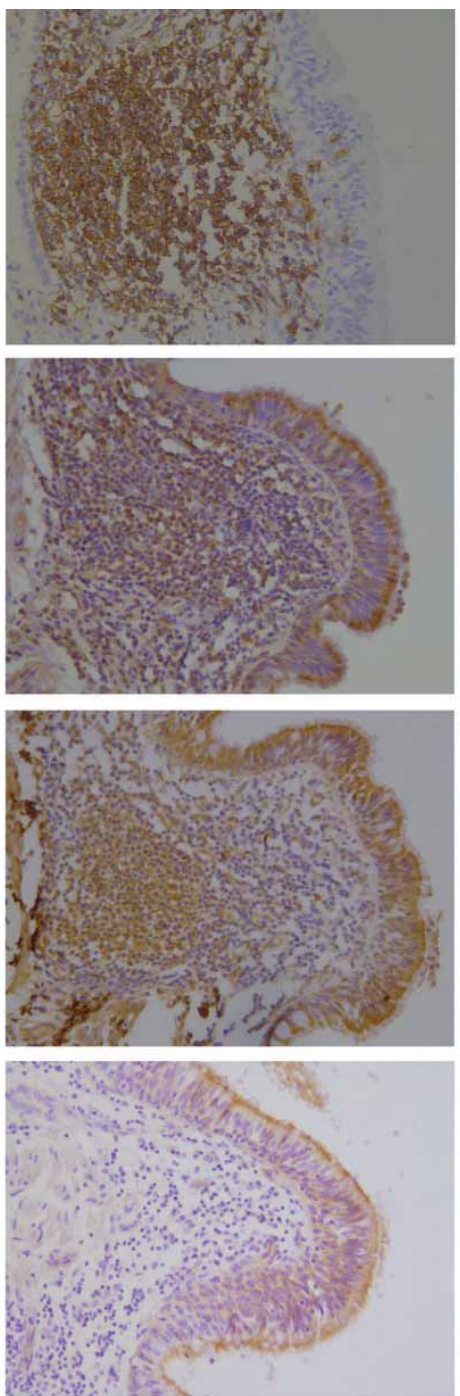

CF
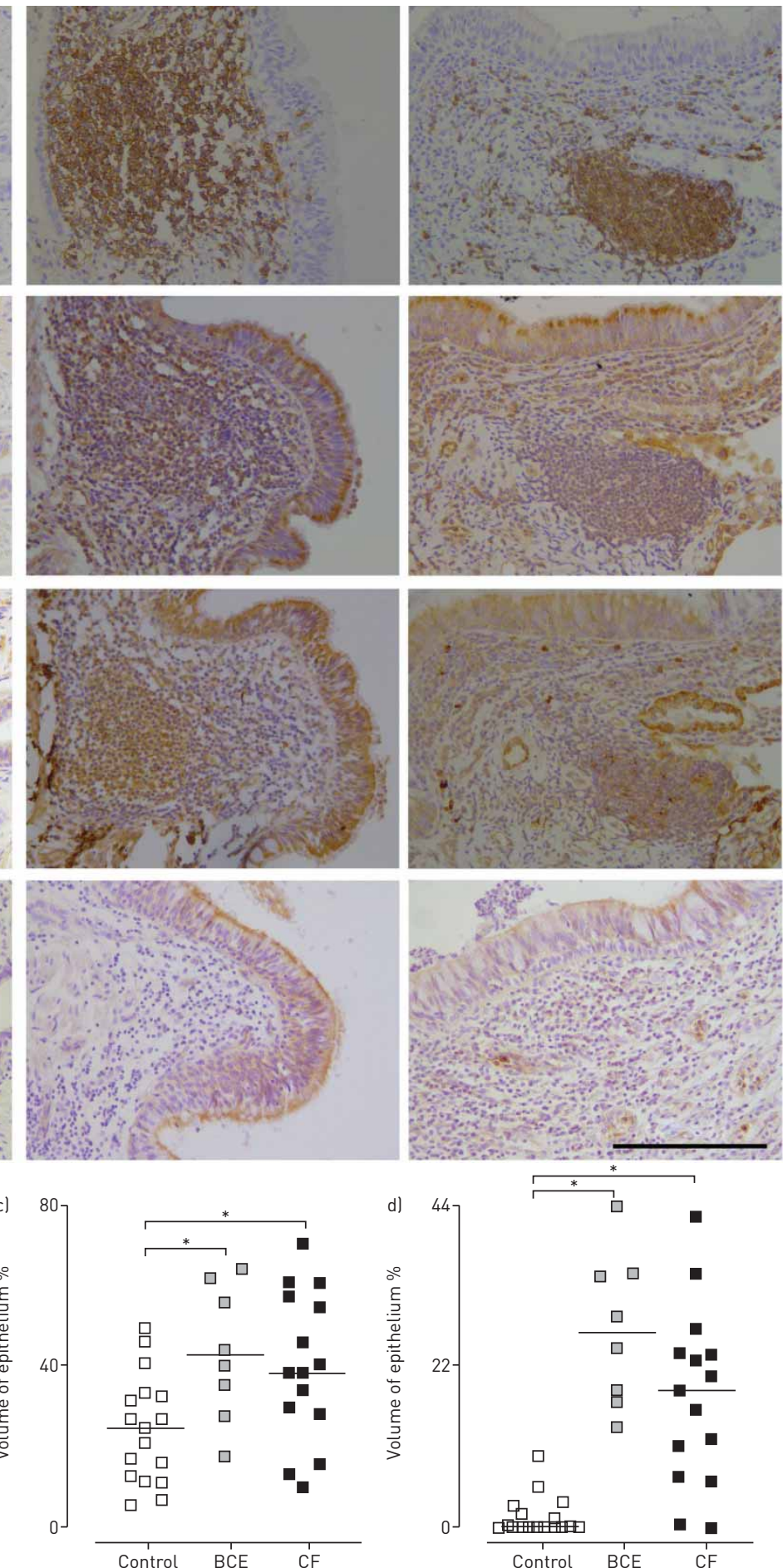

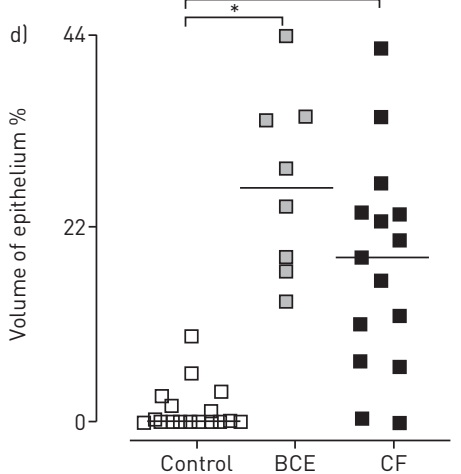

FIGURE 2 Immunostaining for the chemokines CXCL12 and CXCL13 and interleukin (IL)-17A in human lungs. a) Representative photomicrographs. Tissue sections were immunostained for i) CD20, ii) CXCL12, iii) CXCL13 and iv) IL-17A. In controls, only weak staining for CXCL12, CXCL13 and IL-17A was observed in the airway epithelium. In subjects with bronchiectasis (BCE) or with cystic fibrosis (CF), staining for CXCL12 and CXCL13 was strong and localised in CD20 lymphoid aggregates and in the airway epithelium. IL-17A staining was increased in the airway epithelium of subjects with BCE or CF, but was not found in lymphoid aggregates. Scale bar $=100 \mu \mathrm{m}$. Morphometric quantification of epithelial staining for b) CXCL12, c) CXCL13 and d) IL-17A. Tissue sections were immunostained for CXCL12, CXCL13 and IL-17A. Epithelial volume positively stained for CXCL12, CXCL13 and IL-17A and total epithelial volume was measured as described in the methods section. CXCL12, CXCL13 and IL-17A staining percentages were significantly higher in subjects with BCE compared with controls, whereas only CXCL13 and IL-17A immunostaining was significantly higher in CF patients compared with controls. Each symbol represents data obtained from one patient. Horizontal bars represent medians. *: $p<0.05$ compared with controls. 

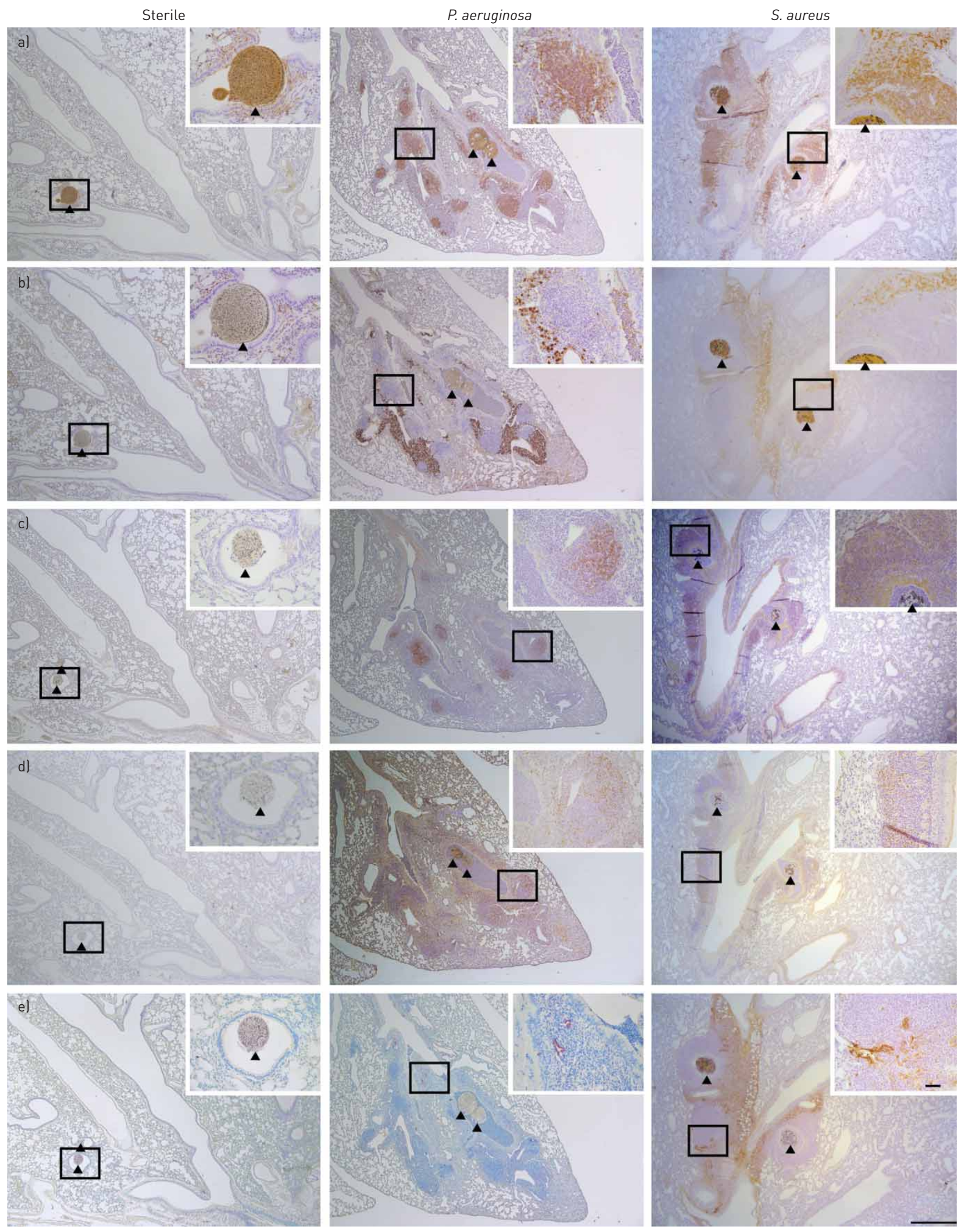

FIGURE 3 Representative photomicrographs depicting the effects of persistent airway infection with Pseudomonas aeruginosa (PAO1) or Staphylococcus aureus (SA) on peribronchial lymphoid neogenesis. Tissue sections were obtained 14 days after intratracheal instillation of sterile, PA01-coated or SA-coated agarose beads. Sections were immunostained (brown) with antibodies to a) B-lymphocytes (CD20), b) T-lymphocytes (CD3), c) follicular dendritic cells (FDCs), d) germinal centres (proliferating cell nuclear antigen) or e) high endothelial venules (peripheral node adressin). In mice infected with PAO1 or with SA, large CD20 ${ }^{+}$lymphoid aggregates were found in the subepithelial area of bead-containing airways; $\mathrm{CD}_{2} \mathrm{O}^{+}$lymphoid aggregates were surrounded by $\mathrm{CD}^{+}$cells and contained FDCs, germinal centres and high endothelial venules, confirming their organisation into tertiary lymphoid organs. By contrast, no lymphoid aggregate was observed at a distance from bead-containing airways in these infected animals. Similarly, no lymphoid aggregate was observed in the lungs of mice instilled with sterile beads. Scale bar $=500 \mu \mathrm{m}$; insert scale bar $=100 \mu \mathrm{m}$. Arrowheads identify beads within the airways. Open boxes identify areas represented in the inserts. 

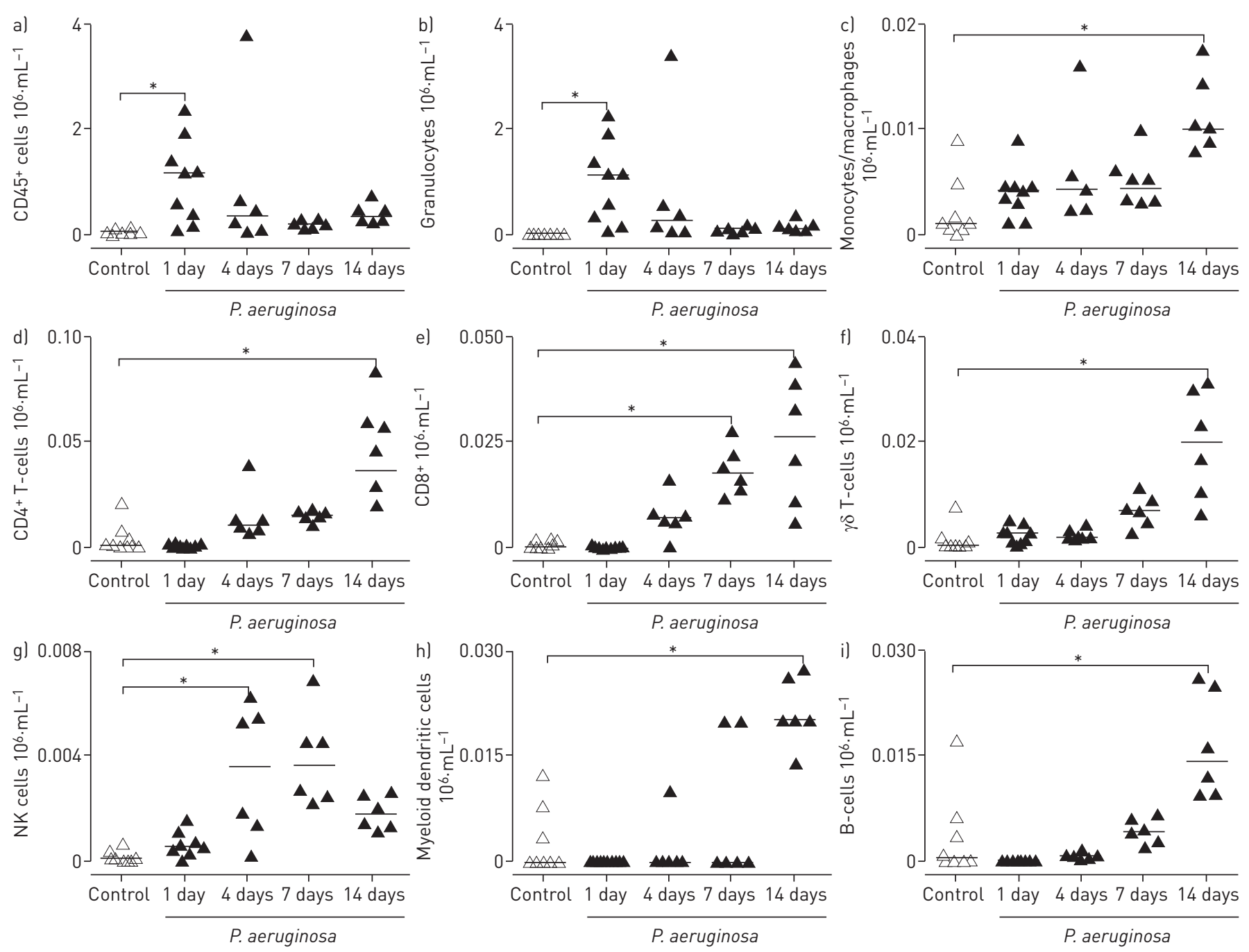

FIGURE 4 Kinetics of airway inflammatory cell recruitment during persistent Pseudomonas aeruginosa (PA01) infection in mice. Mice were infected with PA01-coated beads and bronchoalveolar lavage fluid (BALF) was collected at 1, 4, 7 and 14 days after instillation. Flow cytometry was performed in BALF using markers (see methods and online supplementary table 3) for (a) leukocytes (CD45'), (b) granulocytes, c) monocytes/ macrophages, (d) $\mathrm{CD}^{+}{ }^{\mathrm{T}}$-cells, (e) $\mathrm{CD}^{+} \mathrm{T}$-cells, (f) $\gamma \delta \mathrm{T}$-cells, (g) natural killer cells, (h) myeloid dendritic cells and (i) B-cells. Although the initial response to PA01 infection involved massive recruitment of granulocytes, persistence of infection was associated with a decrease in total number of leukocytes in BALF with concomitant increase in the numbers of B- and T-lymphocytes in the airways compared with control (no instillation) mice. Each symbol represents data obtained from one animal. Horizontal bars represent medians. *: $p<0.05$ compared to controls.

\section{Discussion}

Peribronchial lymphoid aggregates exhibiting characteristics of tertiary lymphoid organs were found in patients with bronchiectasis or with severe $\mathrm{CF}$, while none were observed in control nonsmokers. In mice, persistent (but not transient) bacterial infection with $P$. aeruginosa or $S$. aureus induced rapid recruitment of B-lymphocytes followed by T-lymphocytes around the infected airways. Within 14 days of infection, peribronchial lymphoid aggregates showed structural features that were characteristic of tertiary lymphoid organs. These data suggest that persistent bacterial infection contributes to the lymphoid neogenesis observed in chronic airway diseases.

Lymphoid aggregates containing large numbers of B-cells were found underneath the airway epithelium in all patients with severe CF and in most patients with bronchiectasis. Hubeau et al. [18] studied inflammatory cells infiltrating the airway mucosa in nine CF patients at transplantation compared with three non-CF organ donors and reported large clusters of B-cells in the airway wall in CF patients; in addition, T-cells were increased in distal airways. It was not determined whether these aggregates exhibited the characteristics of tertiary lymphoid organs. WHITwELL [17] performed a histochemical study of surgical specimens obtained in patients with bronchiectasis and described lymphoid aggregates underneath the airway epithelium, without further characterisation. Studies based on bronchial biopsies from CF children [8] or patients with non-CF bronchiectasis [6, 7] reported increased numbers of T-cells $[7,21]$ with only 

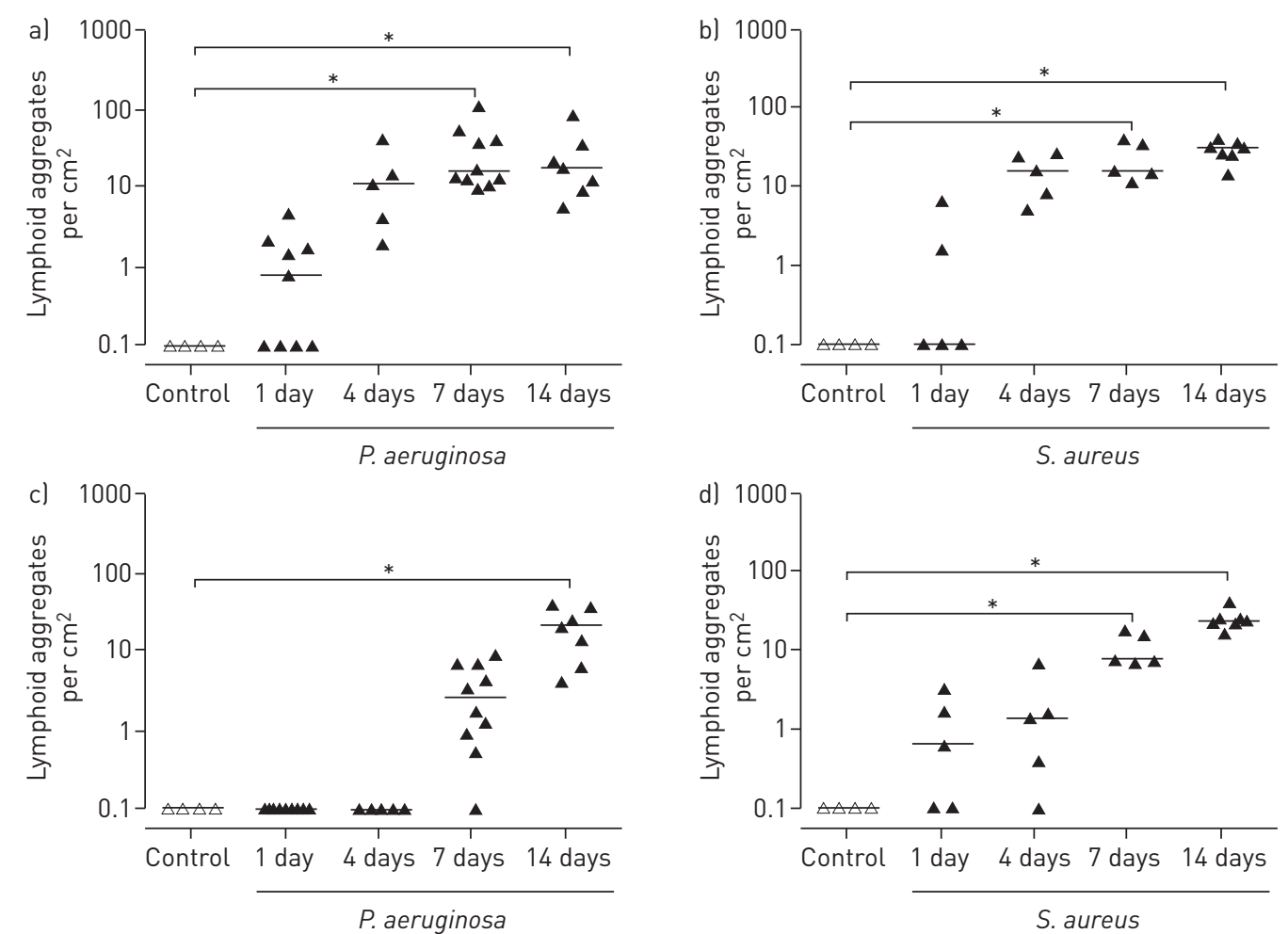

FIGURE 5 Morphometric analysis of $\mathrm{CD}_{20} 0^{+}$and $\mathrm{CD}^{+}$lymphoid aggregates during persistent airway infection with Pseudomonas aeruginosa (PA01) or Staphylococcus aureus (SA) in mice. Mice were infected with PA01- or SA-coated beads and were sacrificed at 1, 4, 7 and 14 days after instillation. Lung sections were immunostained for B-lymphocytes $\left(C D 20^{+}\right)$and T-lymphocytes $\left(C D 3^{+}\right)$. Lymphoid aggregates were counted using morphometric analysis as described in the methods section. No lymphoid aggregate was observed in control (no instillation) mice. Instillation of PA01- or SA-coated beads (solid symbols) resulted in an increase in both $(a, b) \mathrm{CD}_{2} \mathrm{O}^{+}$and $(\mathrm{c}, \mathrm{d}) \mathrm{CD}^{+}$lymphoid aggregates. Each symbol represents data obtained from one animal. Horizontal bars correspond to the median. *: $p<0.05$ compared to controls.

occasional clusters of B-cells just beneath the airway epithelium [24]. We suggest that the small numbers of B-cells in these studies could have been related to a sampling bias as B-cells tend to cluster in specific subepithelial areas, whereas we found that $\mathrm{T}$-cells were more diffusely present underneath the epithelium. Our study confirms that large aggregates of B-cells are present beneath the airway epithelium in patients with bronchiectasis or $\mathrm{CF}$ and extends previous findings by demonstrating that these lymphoid aggregates contained distinct B- and T-cells areas, germinal centres, FDCs and high endothelial venules. These features are the hallmark of tertiary lymphoid organs, in which local adaptive immune response is initiated $[10,11]$.

Using an animal model, we found that persistent airway infection induced peribronchial lymphoid aggregates characterised as tertiary lymphoid organs comparable to those observed in patients with bronchiectasis or CF. Indeed, both P. aeruginosa- and S. aureus-triggered lymphoid aggregates contained germinal centres, FDCs and expressed both CXCL12 and CXCL13. These findings differ from those of a recent study by FLEIGE et al. [25] who reported that repetitive inhalations of heat-inactivated P. aeruginosa induced lymphoid aggregates, which were characterised by the absence of FDCs or other CXCL13-expressing cells in C57BL/6 mice. These differences are probably explained by differences in experimental stimuli and suggest that different stimuli may trigger lymphoid neogenesis via distinct mechanisms. Importantly, our model of infection-induced lymphoid neogenesis largely mimicked expression of CXCL12 and CXCL13 that was found in humans with bronchiectasis or CF. Furthermore, we found that IL-17A, an important player in lymphoid neogenesis [27], was expressed in airway epithelium during persistent bacterial infection in mice. Interestingly, we found that IL-17A was expressed in the airway epithelium of patients with bronchiectasis or with CF, confirming previous data obtained in CF patients [34].

Strengths of the present study rely on the use of human tissues that allowed description of human pathology and mouse models that allowed exploring possible role of persistent bacterial infection in the peribronchial lymphoid neogenesis that was found in humans. Embedding bacteria in agarose beads that were localised within the airway lumen mimicked airway infection in CF airways in which bacteria were localised in mucus plugs within the airway lumens [35]. Localisation of the stimulus in the beads allowed 

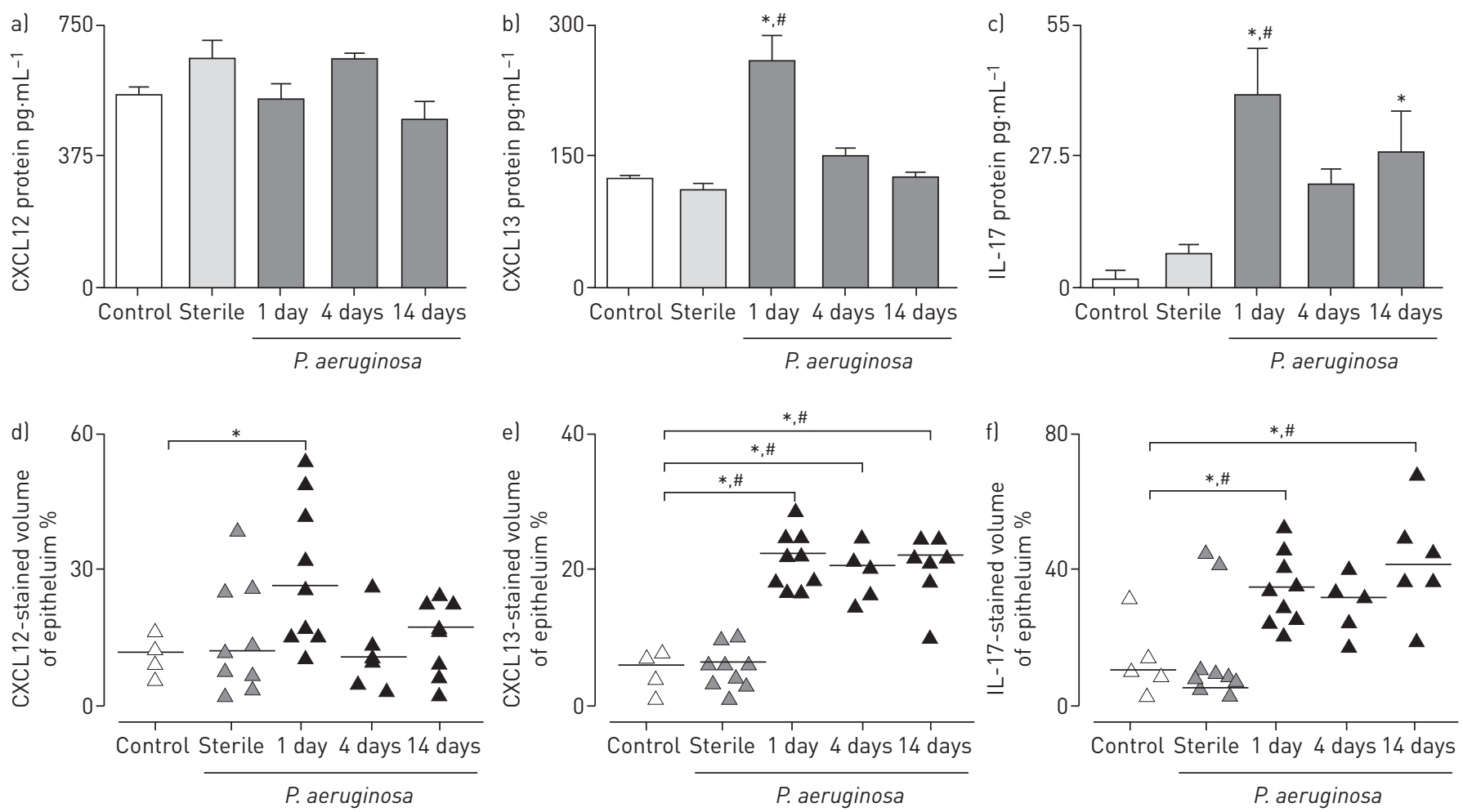

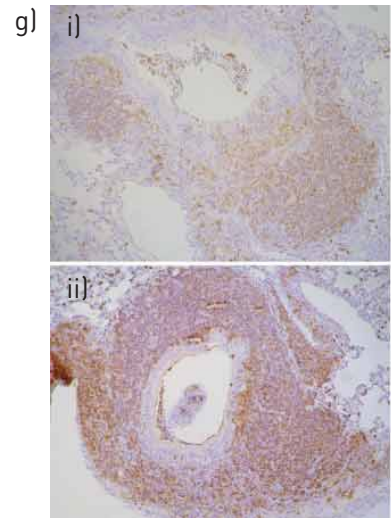

CD20

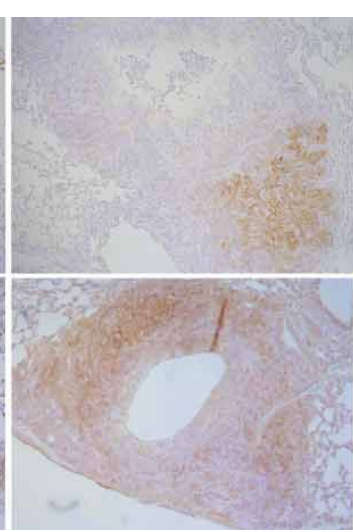

FDC

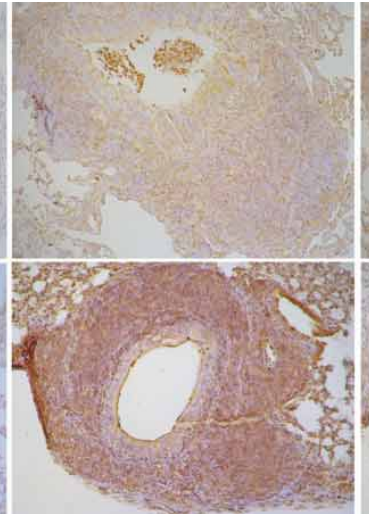

CXCL12

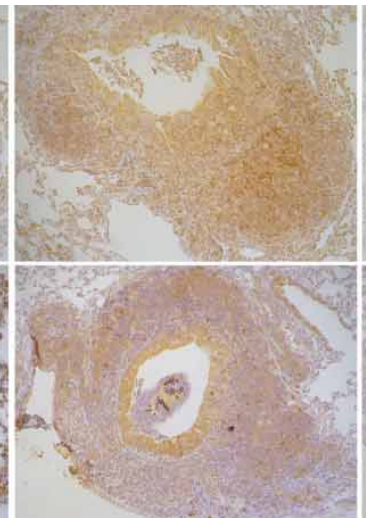

CXCL13

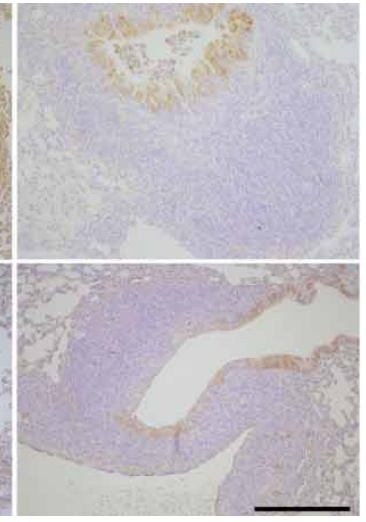

IL-17

FIGURE 6 Expression of CXL12, CXCL13 and interleukin (IL)-17A during persistent bacterial infection in mice. Mice infected with Pseudomonas aeruginosa (PA01)-coated beads were sacrificed at 1, 4 and 14 days after instillation and compared with control (no instillation) and with mice instilled with sterile beads. Because data obtained from control mice or in mice instilled with sterile beads were comparable at all time points, data for control and sterile are presented at a single time point. ELISA measurement of (a) CXCL12, (b) CXCL13 and (c) IL-17A proteins in lung homogenates. Data are presented as mean \pm SEM ( $n=5$ animals per group). ${ }^{*}: p<0.05$ compared with controls; ${ }^{\#}$ : $p<0.05$ compared with sterile. Morphometric analysis of (d) CXCL12, (e) CXCL13 and (f) IL-17A in airway epithelium. Lung sections were immunostained using antibodies directed against CXCL12, CXCL13 or IL-17A and morphometric analysis was performed in epithelium as described in the methods section. Each symbol represents data for one mouse. Horizontal bars represent medians. *: $p<0.05$ compared with controls; ${ }^{\#}$ : p $<0.05$ compared with sterile. g) Representative photomicrographs of chemokine expression in lymphoid aggregate after 14 days of infection by i) PAO1 or ii) Staphylococcus aureus (SA): adjacent lung sections were immunostained using antibodies directed against CD20, follicular dendritic cells (FDC), CXCL12, CXCL13 and IL17A. Immunostainings (brown) showed that CXCL12 and CXCL13, but not IL-17A were expressed in lymphoid aggregates at day 14 after PAO1 or SA infection. IL-17A was strongly expressed by airway epithelium, but not by lymphoid aggregates in both PA and SA infection. Scale bar=200 $\mu$ m.

studies of the spatial relationship between stimulus and lymphoid aggregates, and localisation of lymphoid aggregates adjacent to bead-containing airways suggested a role for airway epithelium in lymphoid neogenesis. This suggestion was further reinforced by the immunolocalisation of CXCL12, CXCL13 and IL-17A in airway epithelium that occurred within $24 \mathrm{~h}$ of bacterial infection. Because infection was triggered using a single intratracheal instillation of bacteria-coated agarose beads, it was possible to examine the kinetics of lymphoid aggregate formation, which is impossible in models that use repetitive inhalation of stimuli [23-25]. For example, single inhalation of heat-inactivated P. aeruginosa failed to 
induce lymphoid follicles in mice, which required repeating weekly inhalation five to 10 times [36], making it difficult to examine kinetics of lymphoid neogenesis in this latter model.

We also recognise limitations to the present study. Human surgical tissues were available only in patients with severe CF at lung transplantation and the presence of peribronchial lymphoid aggregates could not be examined in patients with less severe disease. Similarly, only the subset of patients with localised bronchiectasis undergoing lung resection could be examined. Our study described kinetics of bacteria-induced lymphoid neogenesis but did not examine the roles of homeostatic chemokines (e.g. CXCL12, CXCL13), IL-17A or other molecules (e.g. B-cell-activating factor [37, 38] or IL-6 [39]), which have been implicated in lymphoid neogenesis. Finally, future studies should examine the relative contribution of B-cells and T-cells for triggering lymphoid neogenesis induced by infection.

The present study showed that peribronchial tertiary lymphoid organs were present in patients with advanced (i.e. undergoing surgical resection for localised severe bronchiectasis or lung transplantation) lung disease, whereas a previous study showed that B-lymphocytes were very rare in the lungs of CF fetuses [40], which were presumably exempt from bacterial infection. Because airway infection occurs early in the life of CF patients [41], we speculate that peribronchial lymphoid neogenesis could be an early event in life. Interestingly, studies performed in Scnn $1 b-\mathrm{Tg}^{+}$mice, overexpressing the epithelial sodium channel $\beta$-subunit in airways, found that these mice developed airway mucous plugging, bacterial infection and lymphoid aggregates [42]. Although Scnn $1 b-\mathrm{Tg}^{+}$grown under germ-free conditions were devoid of airway bacterial infection, they still showed airway lymphoid aggregates [42]. However, lymphoid aggregates were found earlier in life and were more prominent when bacterial infection was present in Scnn $1 b-\mathrm{Tg}^{+}$mice [42]. Altogether, these data suggest that dehydration-induced mucus stasis could contribute to promote lymphoid aggregates in airways and that bacterial infection is probably a major driver in the peribronchial lymphoid neogenesis observed in chronic airway diseases.

In summary, our data clearly indicate the presence of a local adaptive immune response involving peribronchial lymphoid neogenesis in bronchiectasis and CF. Importantly, persistent bacterial infection that is also observed in other chronic airway inflammatory diseases (e.g. chronic obstructive pulmonary disease) [43] was sufficient to trigger peribronchial lymphoid neogenesis in mice. Although the present study did not examine the role of lymphoid aggregates in airway defence against pathogens and/or in disease immunopathology [9], our animal model provides a unique model for studying cellular and molecular mechanisms involved in the development of lymphoid aggregates and their persistence in the airways. Future studies should particularly focus on possible roles of airway epithelium in these processes.

\section{Acknowledgements}

We acknowledge the technical contribution of Guiti Thévenot and Mélanie Bigeat (University Paris Descartes, Paris, France) and Abdelhouab Bouaboud and Claire Toustou (Cochin Institute, Paris).

\section{References}

1 Pasteur MC, Bilton D, Hill AT. British Thoracic Society guideline for non-CF bronchiectasis. Thorax 2010; 65: Suppl. 1, i1-i58.

2 Stoltz DA, Meyerholz DK, Welsh MJ. Origins of cystic fibrosis lung disease. N Engl J Med 2015; 372: 351-362.

3 Tang AC, Turvey SE, Alves MP, et al. Current concepts: host-pathogen interactions in cystic fibrosis airways disease. Eur Respir Rev 2014; 23: 320-332.

Chalmers JD, Aliberti S, Blasi F. Management of bronchiectasis in adults. Eur Respir J 2015; 45: 1446-1462. Whitters D, Stockley R. Immunity and bacterial colonisation in bronchiectasis. Thorax 2012; 67: 1006-1013.

Silva JR, Jones JA, Cole PJ, et al. The immunological component of the cellular inflammatory infiltrate in bronchiectasis. Thorax 1989; 44: 668-673.

7 Gaga $\mathrm{M}$, Bentley $\mathrm{AM}$, Humbert $\mathrm{M}$, et al. Increases in $\mathrm{CD}^{+} \mathrm{T}$ lymphocytes, macrophages, neutrophils and interleukin 8 positive cells in the airways of patients with bronchiectasis. Thorax 1998; 53: 685-691.

8 Regamey N, Tsartsali L, Hilliard TN, et al. Distinct patterns of inflammation in the airway lumen and bronchial mucosa of children with cystic fibrosis. Thorax 2012; 67: 164-170.

9 Yadava K, Marsland BJ. Lymphoid follicles in chronic lung diseases. Thorax 2013; 68: 597-598.

10 Pitzalis C, Jones GW, Bombardieri $\mathrm{M}$, et al. Ectopic lymphoid-like structures in infection, cancer and autoimmunity. Nat Rev Immunol 2014; 14: 447-462.

11 Randall TD. Bronchus-associated lymphoid tissue (BALT) structure and function. Adv Immunol 2010; 107: $187-241$.

12 Pabst R, Gehrke I. Is the bronchus-associated lymphoid-tissue (BALT) an integral structure of the lung in normal mammals, including humans. Am J Respir Cell Mol Biol 1990; 3: 131-135.

13 Elliot JG, Jensen CM, Mutavdzic S, et al. Aggregations of lymphoid cells in the airways of nonsmokers, smokers, and subjects with asthma. Am J Respir Crit Care Med 2004; 169: 712-718.

14 Hogg JC, Chu F, Utokaparch S, et al. The nature of small-airway obstruction in chronic obstructive pulmonary disease. $N$ Engl J Med 2004; 350: 2645-2653.

15 Germain C, Gnjatic S, Tamzalit F, et al. Presence of B cells in tertiary lymphoid structures is associated with a protective immunity in patients with lung cancer. Am J Respir Crit Care Med 2014; 189: 832-844.

16 Mansuet-Lupo A, Alifano M, Pécuchet $\mathrm{N}$, et al. Intratumoral immune cell densities are associated with lung adenocarcinoma gene alterations. Am J Respir Crit Care Med 2016; 194: 1403-1412. 
17 Whitwell F. A study of the pathology and pathogenesis of bronchiectasis. Thorax 1952; 7: 213-239.

18 Hubeau C, Lorenzato M, Couetil JP, et al. Quantitative analysis of inflammatory cells infiltrating the cystic fibrosis airway mucosa. Clin Exp Immunol 2001; 124: 69-76.

19 Hwang JY, Randall TD, Silva-Sanchez A. Inducible bronchus-associated lymphoid tissue: taming inflammation in the lung. Front Immunol 2016; 7: 258.

20 Baay-Guzman GJ, Huerta-Yepez S, Vega MI, et al. Role of CXCL13 in asthma: novel therapeutic target. Chest 2012; 141: 886-894.

21 van der Strate BWA, Postma DS, Brandsma CA, et al. Cigarette smoke-induced emphysema: a role for the B cell? Am J Respir Crit Care Med 2006; 173: 751-758.

22 Moyron-Quiroz JE, Rangel-Moreno J, Kusser KR, et al. Role of inducible bronchus associated lymphoid tissue (iBALT) in respiratory immunity. Nat Med 2004; 10: 927-934.

23 Huvenne W, Lanckacker EA, Krysko O, et al. Exacerbation of cigarette smoke-induced pulmonary inflammation by Staphylococcus aureus enterotoxin B in mice. Respir Res 2011; 12: 69.

24 Moghaddam SJ, Clement CG, De la Garza MM, et al. Haemophilus influenzae lysate induces aspects of the chronic obstructive pulmonary disease phenotype. Am J Respir Cell Mol Biol 2008; 38: 629-638.

25 Fleige H, Ravens S, Moschovakis GL, et al. IL-17-induced CXCL12 recruits B cells and induces follicle formation in BALT in the absence of differentiated FDCs. J Exp Med 2014; 211: 643-651.

26 Bracke KR, Verhamme FM, Seys LJ, et al. Role of CXCL13 in cigarette smoke-induced lymphoid follicle formation and chronic obstructive pulmonary disease. Am J Respir Crit Care Med 2013; 188: 343-355.

27 Rangel-Moreno J, Carragher DM, de la Luz Garcia-Hernandez M, et al. The development of inducible bronchus-associated lymphoid tissue depends on IL-17. Nat Immunol 2011; 12: 639-646.

28 Martin C, Thévenot G, Danel S, et al. Pseudomonas aeruginosa induces vascular endothelial growth factor synthesis in airway epithelium in vitro and in vivo. Eur Respir J 2011; 38: 939-946.

29 Burgel PR, Montani D, Danel C, et al. A morphometric study of mucins and small airway plugging in cystic fibrosis. Thorax 2007; 62: 153-161.

30 Rangel-Moreno J, Hartson L, Navarro C, et al. Inducible bronchus-associated lymphoid tissue (iBALT) in patients with pulmonary complications of rheumatoid arthritis. J Clin Invest 2006; 116: 3183-3194.

31 Hsia CC, Hyde DM, Ochs M, et al. An official research policy statement of the American Thoracic Society/ European Respiratory Society: standards for quantitative assessment of lung structure. Am J Respir Crit Care Med 2010; 181: 394-418.

32 Martin B, Auffray C, Delpoux A, et al. Highly self-reactive naive CD4 T cells are prone to differentiate into regulatory T cells. Nat Commun 2013; 4: 2209.

33 Pommier A, Audemard A, Durand A, et al. Inflammatory monocytes are potent antitumor effectors controlled by regulatory CD4 ${ }^{+}$T cells. Proc Natl Acad Sci USA 2013; 110: 13085-13090.

34 Brodlie M, McKean MC, Johnson GE, et al. Raised interleukin-17 is immunolocalised to neutrophils in cystic fibrosis lung disease. Eur Respir J 2011; 37: 1378-1385.

35 Worlitzsch D, Tarran R, Ulrich M, et al. Effects of reduced mucus oxygen concentration in airway Pseudomonas infections of cystic fibrosis patients. J Clin Invest 2002; 109: 317-325.

36 Toyoshima M, Chida K, Sato A. Antigen uptake and subsequent cell kinetics in bronchus-associated lymphoid tissue. Respirology 2000; 5: 141-145.

37 Polverino F, Cosio BG, Pons J, et al. B cell-activating factor. An orchestrator of lymphoid follicles in severe chronic obstructive pulmonary disease. Am J Respir Crit Care Med 2015; 192: 695-705.

38 Seys LJ, Verhamme FM, Schinwald A, et al. Role of B cell-activating factor in chronic obstructive pulmonary disease. Am J Respir Crit Care Med 2015; 192: 706-718.

39 Goya S, Matsuoka H, Mori M, et al. Sustained interleukin-6 signalling leads to the development of lymphoid organ-like structures in the lung. J Pathol 2003; 200: 82-87.

40 Hubeau C, Puchelle E, Gaillard D. Distinct pattern of immune cell population in the lung of human fetuses with cystic fibrosis. J Allergy Clin Immunol 2001; 108: 524-529.

41 Ramsey KA, Ranganathan S, Park J, et al. Early respiratory infection is associated with reduced spirometry in children with cystic fibrosis. Am J Respir Crit Care Med 2014; 190: 1111-1116.

42 Livraghi-Butrico A, Kelly EJ, Klem ER, et al. Mucus clearance, MyD88-dependent and MyD88-independent immunity modulate lung susceptibility to spontaneous bacterial infection and inflammation. Mucosal Immunol 2012; 5: 397-408.

43 Sethi S. Infection as a comorbidity of COPD. Eur Respir J 2010; 35: 1209-1215. 